Gut, 1976, 17, 719-728

\title{
Gastritis duodenitis, and circulating levels of gastrin in duodenal ulcer before and after vagotomy
}

\author{
D. D. Meikle, K. B. TAYloR, S. C. TRUelove, AND R. WhiteheAd \\ From the Nuffield Department of Surgery, the Nuffield Department of Clinical Medicine, and the \\ Department of Morbid Anatomy, Radcliffe Infirmary, Oxford, and the Gastroenterology Division, \\ Department of Medicine, Stanford University Medical Center, California, USA
}

SUMMARY Biopsy specimens have been taken from five standard sites in the stomach and from the duodenal bulb in order to investigate the association of gastritis and duodenitis with duodenal ulcer. Twenty patients with chronic duodenal ulcer were investigated in this manner and in addition had gastric secretion tests and a radio-immune assay of serum gastrin under differing conditions. The patients were then treated either by a truncal vagotomy and pyloroplasty (TVP) or by a highly selective vagotomy without a drainage procedure (HSV). All the investigations were repeated three months postoperatively. Duodenal ulcer was usually associated with gastritis, although this varied in extent and severity from patient to patient. In nearly all the patients, gastritis was present at the pyloric end of the stomach and along the lesser curve. In more than half of the patients, gastritis was also present in the body of the stomach but the fundus was usually spared. Chronic duodenitis was found in the duodenal bulb in all these patients. After vagotomy there was a marked increase in both the extent and severity of the proximal gastritis in both treatment groups but the distal gastritis remain almost unchanged. There was little change in the incidence of duodenitis after vagotomy but its severity was lessened. No correlation was found between the peak acid output (PAO) in response to Histalogand the severity of the gastritis or the duodenitis either before or after operation, with one exception. The postoperative PAO was significantly less in those patients who developed a severe proximal gastritis after vagotomy. No relationship was found between the severity of the distal gastritis and the levels of serum gastrin. No correlation was found between either the basal or peak acid output and the corresponding serum gastrin levels before or after vagotomy.

The gastritis and duodenitis associated with a chronic peptic ulcer have received comparatively little study by the modern method of obtaining multiple biopsy specimens under vision through a fibreoptic endoscope. Our principal object in the present study was to determine the extent and severity of any gastritis or duodenitis in a group of patients with chronic duodenal ulcer who had already been selected for surgical treatment and to determine the effects of two types of vagotomy on the mucosa of the stomach and duodenal bulb. In addition, we set out to determine the levels of circulating gastrin in these patients in response to various stimuli, both before and after operation, and to relate these levels to gastric acid output and to the histological state of the mucosa.

Received for publication 11 June 1976

\section{Methods}

\section{PATIENTS}

Twenty patients attending the Radcliffe Infirmary with chronic duodenal ulcer who had already been selected for surgical treatment were studied shortly before operation and three months postoperatively. Ten of the patients were treated by bilateral truncal vagotomy combined with a Heinicke-Miculicz pyloroplasty (TVP) and the other 10 by a highly selective vagotomy without a gastric drainage procedure (HSV). These two operations were performed at random and the two treatment groups proved to be well matched in terms of age, sex, and length of ulcer history. The majority of the operations were performed by one of us (D.D.M.) and the remainder by two consultant surgeons at the Radcliffe Infirmary. 
BIOPSY OF MUCOSA OF STOMACH AND DUODENAL BULB

Endoscopy was usually performed with an Olympus GIF-D, a forward-viewing instrument through which good-sized biopsy specimens can be obtained. A few examinations were made with an Olympus JFB, a sideways viewing instrument which is highly flexible but suffers from the disadvantage that the biopsy specimens tend to be smaller.

Biopsy specimens were obtained from five standard sites within the stomach and also from the duodenal bulb at least $1 \mathrm{~cm}$ distant from the ulcer (Fig. 1). The specimens were floated off the biopsy forceps into physiological saline and were then immediately transferred to formol saline for fixation. The histological preparation of the specimens was performed by one technician who is solely engaged in dealing with biopsy specimens from the gastrointestinal tract. Multiple specimens were cut from each biopsy specimen and were mounted on three slides, with at least five sections on each slide. One slide from each biopsy specimen was stained by iron haematoxylin and eosin, another by the periodic acid-Schiff method, and the third by a reticulin stain. The slides were examined by one of us (R.W.), who was unaware of the name of the patient, whether the specimen had been taken before or after operation, and to which treatment group the patient belonged.

Gastritis was graded according to the classification of Whitehead et al. (1972) and duodenitis according to that of Whitehead et al. (1975). The grades of gastritis or duodenitis are shown in our subsequent figures according to the key given in Fig. 2.

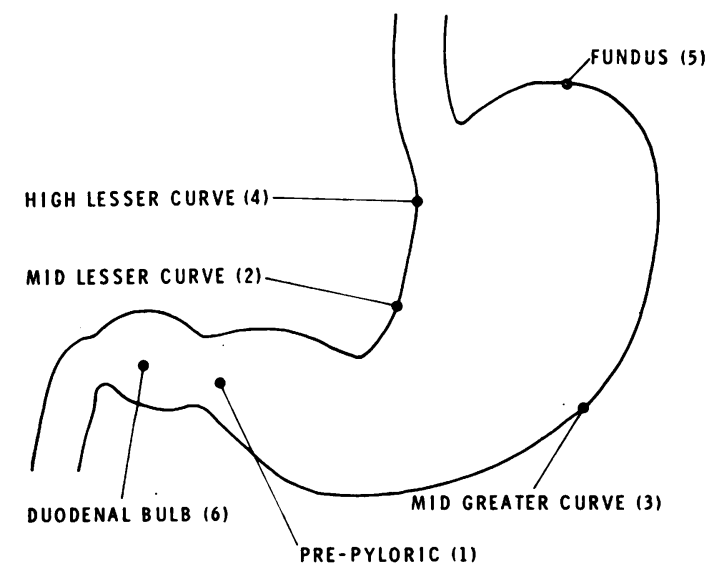

Fig. 1 Standard biopsy sites within the stomach and duodenal bulb. Each site is designated by the number shown.
Gastric Biopsies

Normal Mucosa

Superficial Gastritis

Mild Atrophic Gastritis

Moderate

Atrophic Gastritis

?? Unsatisfactory Biopsy

Fig. 2 Key to Figs. 3, 4, and 5.

\section{GASTRITIS INDEX}

To facilitate comparison of the different histological pictures found in the various biopsy specimens, each grade of gastritis was given a number. Normal mucosa was designated 0 , superficial gastritis 1 , mild atrophic gastritis 2 , moderately severe atrophic gastritis 3, and severe atrophic gastritis 4 . As the histological abnormalities in the different grades of gastritis appear to be progressively more severe from grades 1 to 4 it seems reasonable to score each biopsy according to its grade as an index of the severity of gastritis. Then, for example, each biopsy specimen showing superficial gastritis scores 1 while a specimen with severe atrophic gastritis scores 4 . A gastritis index for the whole stomach or for the antrum or the body can then be obtained by summing the appropriate biopsy indices.

\section{DUODENITIS INDEX}

In a similar way grades of duodenitis found in the duodenal bulb biopsy specimens were given an index of 1 for mild duodenitis, 2 for moderately severe duodenitis, and 3 for severe duodenitis. Normal mucosa was scored 0 .

\section{GASTRIC ACID OUTPUT}

Gastric acid output was estimated both before and after operation in all patients by gastric secretion tests which were performed in the morning after an overnight fast. A Salem size 14 French gauge nasogastric sump tube was passed into the stomach and the patient positioned horizontally, lying on the left side. The position of the tube was checked by means of the water test (Findlay et al., 1972). Resting contents were aspirated and discarded and 15minute collections of gastric juice were then made by frequent, intermittent hand aspiration using a $20 \mathrm{ml}$ syringe. After a basal hour, betazole hydrochloride (Histalog), 1.7 mg per $\mathrm{kg}$, was injected subcutane- 
ously and a further eight 15 -minute collections were made. During the test blood samples were collected intermittently for estimations of serum gastrin as will be described.

The Histalog gastric secretion test was repeated three months after operation. In addition, at this time, all the patients agreed to have an insulin test. The same technique was used for collecting the gastric juice but soluble insulin, in a dose of $\mathbf{0 . 2}$ units per $\mathrm{kg}$ body weight, was given intravenously instead of Histalog. Blood samples were collected during the basal hour and at 30,45 , and 60 minutes after the insulin injection for blood sugar measurements. A fall in blood glucose level below $40 \mathrm{mg}$ / $100 \mathrm{ml}(2 \cdot 2 \mathrm{mmol} / \mathrm{l})$ was achieved in all cases.

\section{Analysis}

After noting the volume, $1 \mathrm{ml}$ of each 15 -minute sample of gastric aspirate was titrated with $0 \cdot 1 \mathrm{~N}$ $\mathrm{Na} \mathrm{OH}$ to a $\mathrm{pH} 7$ end-point using a micro-electrode pH meter. The total acid output of each 15-minute sample was expressed in mmol.

Basal acid output (BAO) was calculated for the whole hour by addition of the four quarter-hour samples. The peak acid output (PAO) after Histalog or insulin was calculated by adding the two highest consecutive 15-minute outputs and multiplying by two. In addition, the insulin tests were assessed by the criteria of Hollander (1948).

\section{BLOOD SAMPLING FOR SERUM GASTRIN} STUDIES

Blood samples $(10 \mathrm{ml})$ for serum gastrin estimations were drawn from an indwelling tapped Teflon cannula which was positioned in an arm vein at the beginning of the test and retained until the last sample had been drawn.

During the gastric secretion tests the first venous blood sample was taken half an hour before the Histalog or insulin was administered. After Histalog, four additional venous blood samples were taken at half-hour intervals and, after the insulin, usually eight further samples at quarter-hour intervals.

In addition, fasting and postprandial blood samples for gastrin assay were collected on a separate morning both before and three months after operation. The patients were asked to fast from midnight and to come to the hospital by 9 a.m. A fasting blood sample was taken and they were given a breakfast. At intervals of $\mathbf{3 0}$ minutes, timed from when the breakfast was started, four additional venous blood samples were collected. In most cases

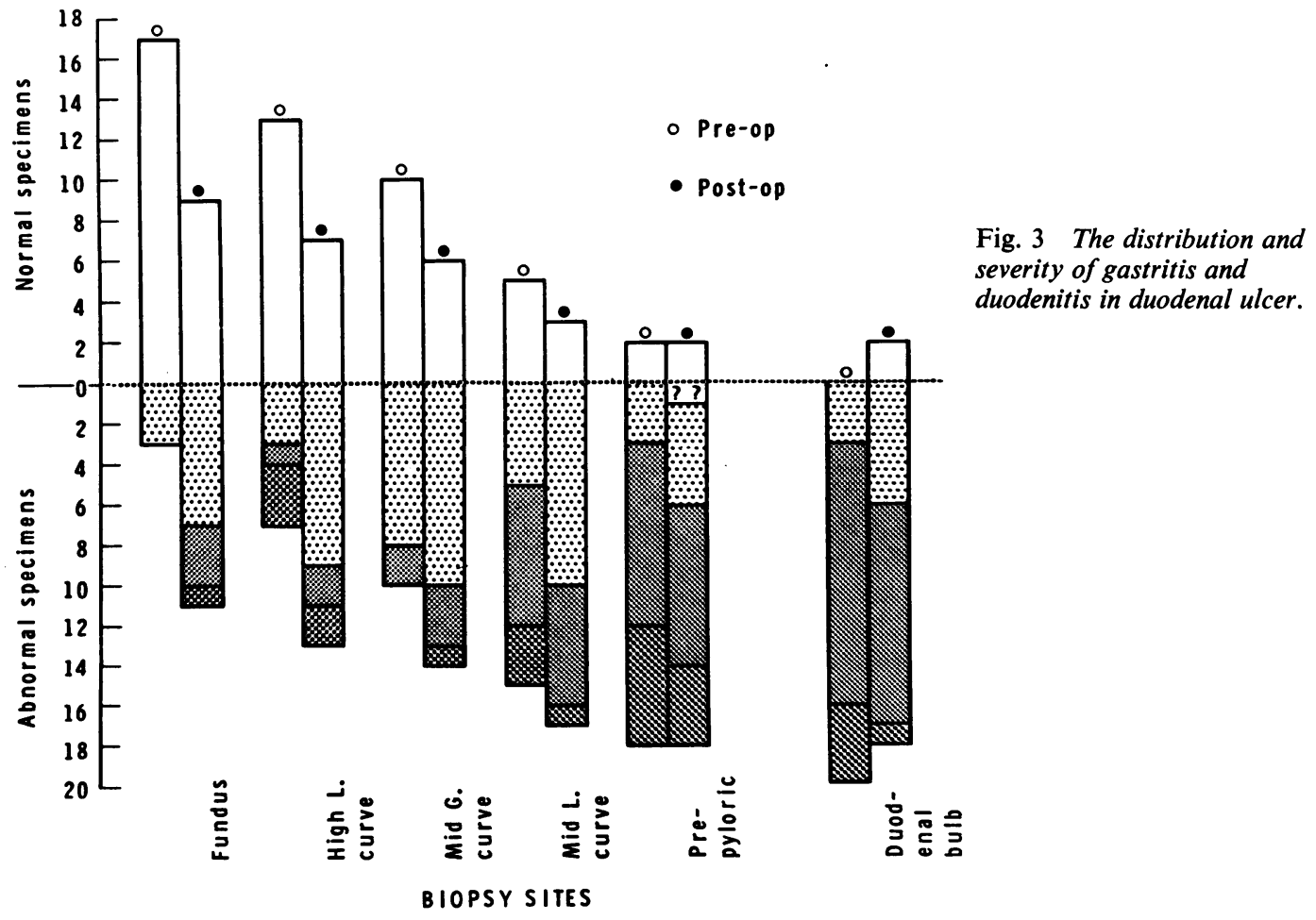


the breakfast consisted of cereal with milk and sugar, bacon and egg, toast, and tea or coffee. Occasionally, however, the patient was not able to eat this full breakfast and had a more limited meal. However, each patient had a similar breakfast after operation to the one taken before operation.

After each of the different tests the blood samples were centrifuged and the serum separated and stored in Bijou bottles in a deep freeze at minus $20^{\circ} \mathrm{C}$. Each specimen was given a code number and at intervals of two to three months the samples were collected together in an insulated box containing dry ice and dispatched by air freight to California for the radioimmunoassay. As other samples of serum with similar styled code numbers from patients with other diseases were also included, the results of the radio-immuno assay were not biased by any knowledge of the type of specimen.

ESTIMATION OF SERUM GASTRIN ACTIVITY This was carried out in the Department of Medicine, Stanford University Medical Center, by a modification of the radio-immunoassay method of Yalow and Berson (1970).

Antisera to crude procine gastrin conjugated with bovine-serum albumin by the method of Yip and Jordan (1970) were obtained by immunization of Hartley-strain female guinea-pigs according to a schedule of multiple subcutaneous injections at one month intervals. These antisera have been characterized by Rosenquist and Holmquist (1974). They recognize the $\mathrm{C}$-terminal tetrapeptide of synthetic human gastrin I (ICI) and display increasing binding over the tetraoctapeptide range.

Synthetic human gastrin I (ICI Batch No. 630/20) labelled with 125 iodine by the modified chloramine$T$ method of Hunter and Greenwood (1962) was used as the trace. Antisera concentrations were selected to give a maximal binding capacity of approximately $30 \%$. All serum samples were tested in triplicate, with duplicate controls (no antibody added). Separation of antibody-bound to antibodyfree gastrin was effected by adding an equal volume of polyethylene glycol 6000 (PEG), 30\% wt/wt solution in distilled water to the reaction mixture. Standard inhibition curves were established for each assay.

Radioactivity in the PEG precipitates was estimated in an automatic gamma counter (Nuclear Chicago Model 1035, Serial 130). The counts were transmitted by teletype to a computer and the computer data expressed as means \pm one standard deviation, a standard curve being obtained for the same assay. Our own observations have established a normal range of $0-200 \mathrm{pg} / \mathrm{ml}$ for human subjects under fasting conditions.
Results

EXTENT AND SEVERITY OF GASTRITIS AND DUODENITIS ASSOCIATED WITH CHRONIC DUODENAL ULCER BEFORE AND AFTER VAGOTOMY

Figure 3 shows the histological findings in the biopsy specimens taken from the six standard sites in each patient both before and three months after vagotomy. It can be seen that the fundus of the stomach was normal in $17(85 \%)$ of the 20 patients with duodenal ulcer before operation. In only three $(15 \%)$ were the fundal biopsy specimens abnormal and they showed superficial gastritis only. The percentage of normal biopsy specimens decreased as the site of biopsy progressed down the stomach from the proximal fundal to the distal pre-pyloric site and, in addition, the more severe grades of gastritis were found distally. Thus it is seen that duodenal ulcer is usually associated with gastritis, although this varies in extent and severity from patient to patient. In the majority there is gastritis involving the pre-pyloric region and mid-lesser curve. The stomach proximal to this, corresponding to the body gland area, is only occasionally involved. The gastritis is not only much more frequent in the distal stomach but it is also more severe.

The duodenal bulb biopsy specimens all showed an increased chronic inflammatory cell infiltrate of the lamina propria and this was of moderate or severe degree in $17(85 \%)$. Thus we can conclude that duodenal ulceration is always associated with duodenitis. Furthermore, as the biopsy specimens were taken from an area of the bulb well clear of the ulcer, the duodenitis is at least a regional manifestation and not purely localized to the immediate vicinity of the ulcer.

After treatment by either form of vagotomy there was a decrease in the number of normal specimens at each site except at the pre-pyloric site where there was no change. In addition, more of the biopsy specimens showed atrophic gastritis at the fundal and mid-greater curve biopsy sites. When the indices of gastritis found preoperatively at the different biopsy sites were compared with those found after operation, the postoperative deterioration at the fundus was highly significant $(P<0.01)$ and that at the mid-greater curve site significant $(P<0.05)$. At the two distal sites, the mid-lesser curve and the prepyloric, the changes were not statistically significant. In specimens from the duodenal bulb, however, there was some improvement postoperatively in the incidence and severity of the duodenitis.

Similar postoperative changes in the picture of gastritis and duodenitis were seen regardless of the particular form of vagotomy (Figs. 4 and 5). In 


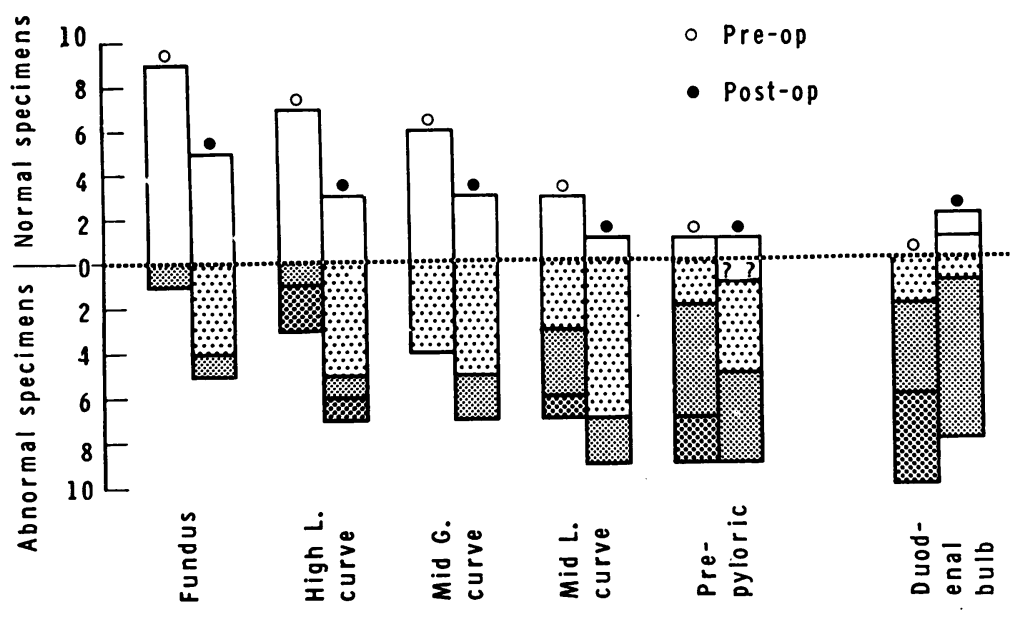

BIOPSY SITES

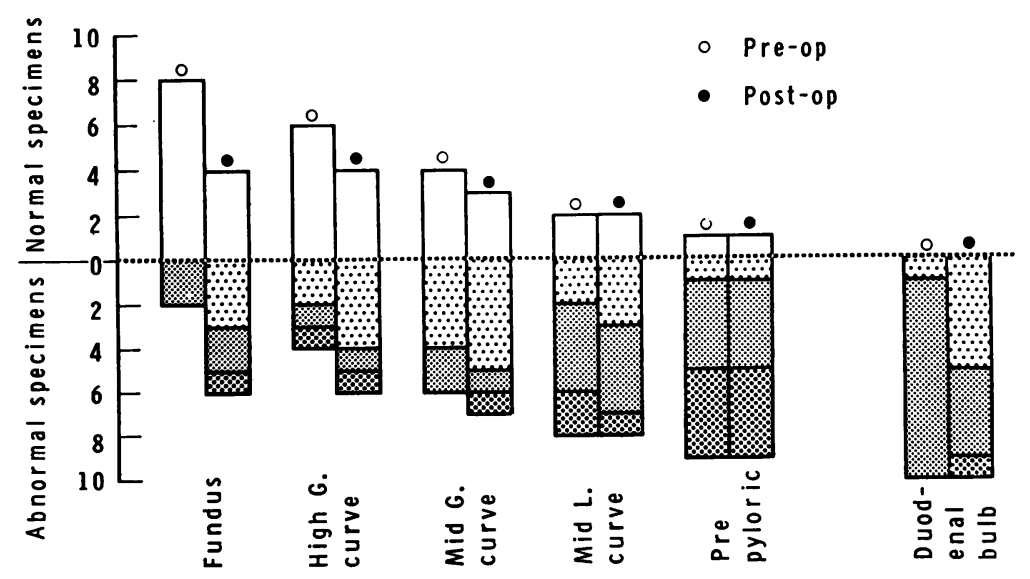

BIOPSY SITES
Fig. 4 The distribution and severity of gastritis and duodenitis in duodenal ulcer before and after HSV.
Fig. 5 The distribution and severity of gastritis and duodenitis in duodenal ulcer before and after TVP. each group there was an improvement in the duodenitis but an increase in both the incidence and severity of the gastritis affecting the proximal stomach (corresponding to the body and fundus) with atrophic gastritis developing in the fundus after operation in several patients, whereas none showed this before operation. After TVP, there was greater improvement in the duodenitis, less atrophy at the fundus, an improvement in the severity but not in the incidence at the pyloric end of the stomach, and an increased incidence of gastritis at the middle of the lesser curve. When the two distal biopsy sites were considered together as a measure of antral gastritis, there was very little difference between the two post-vagotomy groups, although there was a slight overall worsening in the TVP group. These postoperative differences between the two treatment groups, which were well matched preoperatively, are not statistically significant. However, each group consisted of only 10 patients and it would be advisable to study a larger number before deciding that there is no difference between the two operations in this respect.

\section{GASTRIC ACID OUTPUT BEFORE AND AFTER VAGOTOMY}

In Fig. 6 the pre- and postoperative acid output values are shown. It can be seen that both operations produced a highly significant reduction of the PAO in response to Histalog stimulation. In addi- 


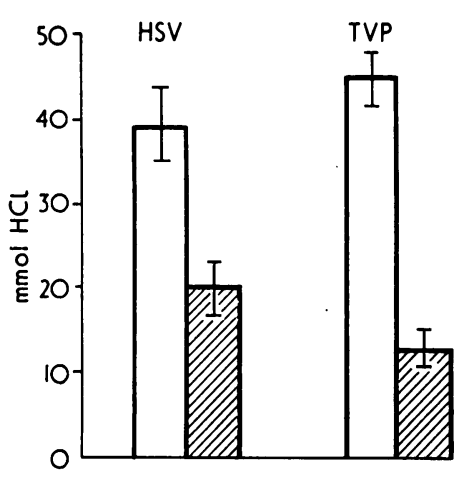

Fir. 6 Peak acid output after Histalog stimulation before and after the two types of vagotomy.

Preoperative. $\mathbb{Z}$ Postoperative. 王SE mean.

tion, there was no statistically significant difference between the PAO of patients in the post-TVP group when compared with the peak PAO of patients in the post-HSV group. Likewise, the PAO after insulin stimulation three months postoperatively was similar in the two treatment groups. It can be concluded that both operations produce similar highly signifcant reductions of the peak acid output.

\section{CORRELATION OF SEVERITY OF GASTRITIS}

WITH GASTRIC ACID OUTPUT

Mucosal biopsy specimens taken from the fundus, high lesser curve, and the middle of the greater curve almost always consist of body mucosa. As these biopsies were taken from widely separated areas of the corpus it seems reasonable to sum the individual indices of gastritis of these three proximal biopsies as a representative index of the gastritis affecting the body of the stomach, the Proximal Gastritis Index (PGI). Similarly, the sum of the indices of gastritis for the distal two biopsy sitesnamely, from the middle of the lesser curve and the pre-pyloric biopsy site-has been used as a representative index of the gastritis involving the antrum, the Distal Gastritis Index (DGI). For an index of gastritis affecting the stomach as a whole, the sum of the indices from all five biopsy sites, has been used and termed the Overall Gastritis Index (OGI).

In order to investigate a relationship between gastritis and gastric acid secretion, the patients have been put into different groups according to the value of the indices of gastritis in the two main areas of the stomach and in the stomach as a whole. The acid outputs of the patients having the lowest indices of gastritis have been compared with those of patients having the highest indices of gastritis by Student's $t$ test. There were no statistically signifi- cant differences between the PAOs of the different gastritis groups, either before or after operation, with one notable exception-namely, that there was a highly significant difference between the PAO (Histalog) of patients in the lowest and highest PGI groups after vagotomy $(P<0.001)$. As no significant differences were detected between the acid output of patients with the highest gastritis indices and that of patients with the lowest gastritis indices, whether these were for the proximal stomach only, the distal stomach only, or the whole stomach, it is concluded that the gastritis in duodenal ulcer patients does not affect the level of acid output after Histalog stimulation.

However, after treatment of the duodenal ulcer by vagotomy, the PAO (Histalog) was much lower in patients with the highest proximal gastritis indices than in those with only minimal proximal gastritis. By contrast, the postoperative PAO did not vary with the Distal Index nor with the Overall Gastritis Index.

When the acid output of patients having the lowest indices of duodenitis was compared with the acid output of those with the highest indices, no significant differences were detected between the different histological groups both before and after vagotomy. Thus it is concluded that the degree of duodenitis also is not related to the acid output of the stomach.

SERUM GASTRIN LEVELS

The Table and Fig. 7 show the mean fasting and post-prandial levels of serum gastrin in the patients in the two treatment groups, both before and after operation. It can be seen that all the mean levels were lower in the HSV group but, in fact, the differences were not significant when compared by Student's $t$ test. There were considerable variations in the serum of gastrin levels under the same conditions from patient to patient. However, in both groups there was a marked rise in the level of serum gastrin after food both before vagotomy and also after either form of vagotomy. Furthermore, neither form of vagotomy produced significant alterations of either the fasting or post-prandial levels of serum gastrin.

The mean fasting gastrin values, based on two samples, in the 10 subjects before and three months after truncal vagotomy and drainage were $164 \mathrm{pg} / \mathrm{ml}$ and $108 \mathrm{pg} / \mathrm{ml}$. By Student's $t$ test these are not significantly different. Comparable figures for the highly selective vagotomy patients were 125 and 147 $\mathrm{pg} / \mathrm{ml}$. There is no significant difference between the somewhat depressed post-TV and the somewhat elevated post-HSV mean values. There is wide variation of the serum gastrin levels under the same conditions from patient to patient, however. Therefore 
HSV

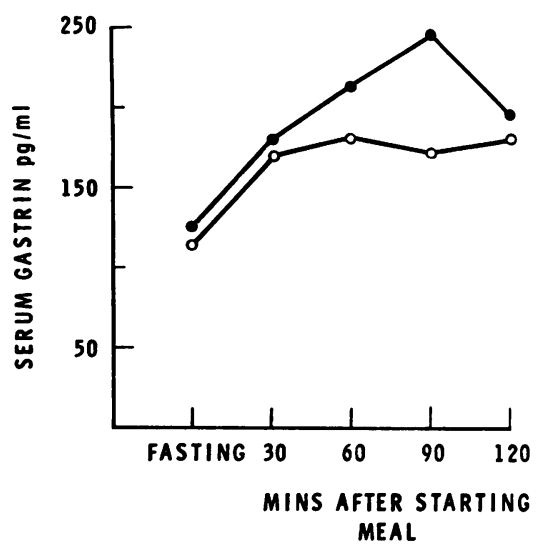

TVP

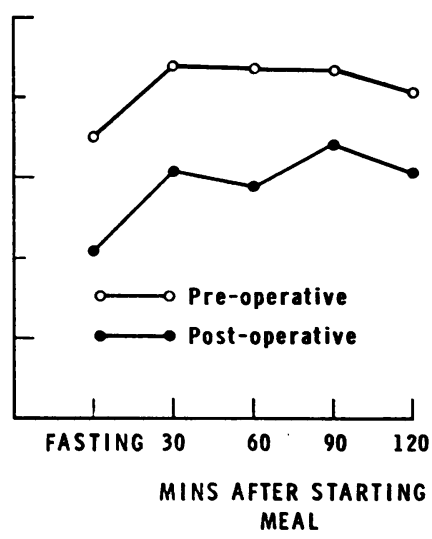

Fig. 7 Fasting and post-prandial levels of serum gastrin before and after the two types of vagotomy.

Table Fasting serum gastrin levels $(\mathrm{pg} / \mathrm{ml})$ before and after two types of vagotomy

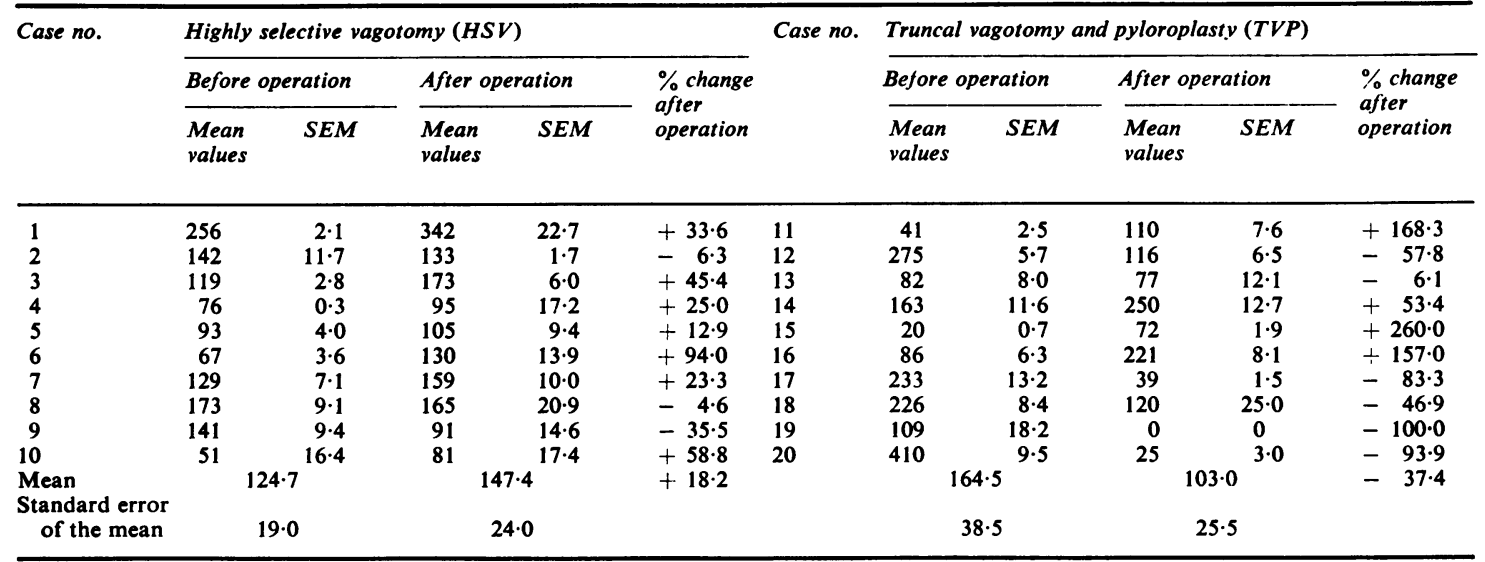

we examined the percentage increase or decrease of fasting serum gastrin for each subject after surgery. Again no significant change was seen in either operative group.

After food a marked rise in serum gastrin occurred in all subjects in each group, pre- and postoperatively (Fig. 7). The means of the summated serum gastrin levels for the HSV group were $181 \mathrm{pg} / \mathrm{ml}$ before and $215 \mathrm{pg} / \mathrm{ml}$ after surgery and the comparable figures in the TV group were $202 \mathrm{pg} / \mathrm{ml}$ and $161 \mathrm{pg} / \mathrm{ml}$. There are no significant differences between the pre- and postoperative results in each group nor between the two groups.

Serum gastrin levels were also estimated during the Histalog tests and the postoperative insulin test. The mean values of the serum gastrin for the two treatment groups during the Histalog test were found to be well matched both before and after operation and also it was found that neither type of vagotomy produced a significant alteration of the different mean serum gastrin values when assessed by Student's $t$ tests. Similarly, when the corresponding insulin-test serum-gastrin levels of the two postvagotomy groups were compared by $t$ test, there were no significant differences between them. However, when the basal serum gastrin levels were compared with the highest post-insulin levels, highly significant differences were found in the post-HSV group $(P<$ $0.02)$ but not in the post-TVP group (P $>0 \cdot 1)$.

\section{GASTRITIS AND SERUM GASTRIN LEVELS}

The effect of gastritis on the release of gastrin from the stomach into the circulation has been assessed by comparing the serum gastrin levels of patients with the most severe grades of gastritis with the serum gastrin levels of those with the lowest grades of 
gastritis. As there were no significant differences between the corresponding serum gastrin levels of the two treatment groups, the two groups have been combined for this purpose. No statistically significant differences were found between the levels of serum gastrin of the patients in the lowest and highest groups of PGI or DGI or OGI, either before or after operation.

\section{DUODENITIS AND SERUM GASTRIN LEVELS}

In a similar way, the relationship between the severity of duodenitis and the serum gastrin has been investigated by comparing the corresponding levels of serum gastrin of each of the patients in the lowest duodenitis indices group with that of each of the patients in the highest duodenitis indices group by Student's unpaired $t$ test. Again no significant differences were found and it is concluded that the serum gastrin levels of patients with severe duodenitis of the duodenal bulb associated with duodenal ulcer are not different from those patients with only mild duodenitis and the same is true for these patients after vagotomy.

\section{COMPARISON OF ACID OUTPUT WITH}

SERUM GASTRIN LEVELS

To determine whether there is a correlation between the serum gastrin levels and the acid output the coefficients of linear correlation were determined using the Bravais-Pearson formula programmed into a computer. It was found that there were no significant correlations between the levels of serum gastrin in the samples taken during the basal hour of the preoperative Histalog test, the postoperative Histalog, or postoperative insulin tests and the respective BAO values of the two treatment groups, with one exception. This occurred in the post-TVP group, during the insulin test, where the $r$ value was $0 \cdot 8$. When the different highest gastrin levels after Histalog or insulin were correlated with the corresponding PAO values of patients in the different treatment groups, the $r$ values were all low. This lack of correlation is probably because the serum gastrin is only one of several variables in the control of gastric acidity. Also, the serum gastrin levels fluctuate widely from time to time and blood samples for assay were only collected at half-hourly intervals. In this study, no attempt was made to correlate the serum gastrin level with rates of acid secretion in individual patients.

\section{Discussion and conclusions}

The question of the association between chronic peptic ulcer and gastritis remains controversial. In the past, the methods of study have been defective.
Post-mortem material is useless for studying gastric pathology unless very special steps are taken to prevent autolysis (Faber, 1935). The study of resected portions of stomach suffers from the limitation that histological changes in the mucosa occur during the course of the operation (Schindler et al., 1939; Sanders and Mecray, 1941). Blind biopsy was a useful step forward but usually the specimen came from only one site in the stomach, although there was the advantage that serial studies were possible.

Modern fibreoptic endoscopy has transformed the situation. The whole of the upper gastrointestinal tract as far as the descending duodenum can be visualized and biopsy specimens obtained under vision. The method is new and so far has not been employed very much for scientific investigation although widely used as a diagnostic tool.

In the present study, biopsy specimens have been obtained from five sites within the stomach and also from the duodenal bulb. In addition, the gastric acid secretion and circulating levels of gastrin have been measured in every patient. All these observations were performed initially on patients with duodenal ulcer who had been selected for surgical treatment. Half the patients were treated by a truncal vagotomy and pyloroplasty (TVP) and half by the new operation of highly selective vagotomy. Three months after the operation, the various investigations were all repeated.

Gastritis was present in nearly all these patients with duodenal ulcer but it tended to be concentrated and to be most severe in the distal part of the stomach - namely, in the antrum and along the lesser curve. More than half of the patients showed some degree of gastritis in the body of the stomach but the fundus was usually spared. The findings contrast with what is found in gastric ulcer, in which widespread gastritis is a common finding (Gear et al., 1971).

The effect of vagotomy on the histological picture was similar whichever type of vagotomy was performed. After TVP and HSV alike, the gastritis in the pyloric end of the stomach tended to lessen, whereas it became worse in the more proximal areas. It is especially noteworthy that, after vagotomy, the fundus was frequently affected by gastritis, whereas it was exceptional for it to be affected beforehand. Moreover, the postoperative fundal gastritis was frequently atrophic and not merely superficial.

Two points are worthy of discussion in this context. The first is that it is possible that the proximal gastritis which develops after a vagotomy may be a progressive change which will eventually lead to severe atrophic gastritis in a large proportion of the patients. This would explain previous findings that 
intrinsic factor secretion and vitamin $\mathrm{B}_{12}$ absorption are diminished after vagotomy (Cox et al., 1964; Bitsch et al., 1966; Adams et al., 1967). There is also the possibility that atrophic gastritis is a precancerous lesion. Both megaloblastic anaemia and cancer of the stomach are late complications of partial gastrectomy, after which gastritis is very common in the gastric stump (Stalsberg and Taksdal, 1971). It is often supposed that a vagotomy is far less likely to be followed by widespread gastritis but the present results cast doubt on this view. It appears to be highly desirable that the patients included in the present study should be re-examined by endoscopy with biopsy after a further interval of time to determine if the gastritis has continued to progress.

The second point concerns the mechanism for the development of gastritis postoperatively in areas of the stomach previously healthy. If the function of the pylorus has been disrupted by a pyloroplasty, it is easy to see that reflux of duodenal contents may readily occur and might be responsible for causing the gastritis. However, in the present series, patients treated by highly selective vagotomy, in which the pylorus is left untouched and the vagal nerve supply to the antrum and duodenum is preserved, showed a worsening of the gastritis in the proximal stomach similar to that found after TVP. This suggests that the pyloroplasty is not relevant to the proximal gastritis, and, by a process of exclusion, one is driven to conclude that the vagotomy is responsible. However, we are totally ignorant of the mechanisms whereby division of the vagal nerve could induce mucosal disease. It is possible that the gastritis is the result of trophic changes after the vagotomy but, at the present time, this is no more than speculation.

There was no correlation between the severity of the distal gastritis, even with atrophy, and the levels of gastrin in the serum. Whether this means that the size and function of the antral gastrin-secreting cell population is unaffected by the antral gastritis associated with peptic ulcer disease or that change in the one is compensated for by inverse change in the other can be resolved only by quantitative determinations of the antral G-cell mass.

Duodenitis appears to be an invariable accompaniment of chronic duodenal ulcer, as judged by the present series. Although only the duodenal bulb was biopsied, care was taken to obtain the biopsy specimen well away from the actual ulcer. After vagotomy, the duodenitis frequently improves. It is possible that the sharp reduction in the acid and pepsin output of the stomach brought about by vagotomy is responsible for the alleviation of the duodenitis, although, if this is the explanation, it makes the worsening of the gastritis in all the proximal areas of the stomach even more mysterious.
A further reason for doubting whether the improvement in the duodenitis which occurs after vagotomy can be attributed to the reduction in the acid output of the stomach is that the degree of duodenitis before operation is not correlated with the acid output of the stomach.

After a vagotomy, whether it be highly selective or truncal, the fasting levels of serum gastrin show no significant change from preoperative levels. Furthermore, preoperative levels do not differ from those in a healthy control group of subjects, a finding in accord with those of Trudeau and McGuigan (1970) and Wesdorp and Fischer (1974) but different from that of Becker et al. (1973), who found significantly higher fasting serum-gastrin levels in patients with duodenal ulcer compared with control subjects.

McGuigan and Trudeau (1972) reported four patients who were treated by vagotomy and antrectomy for duodenal ulcer and in whom postoperative fasting serum-gastrin levels were markedly reduced, almost certainly due to removal of a major source of gastrin secretion. These authors found no significant change in fasting gastrin levels in 24 duodenal ulcer patients after truncal vagotomy and pyloroplasty, however, although the rates of gastric acid secretion were much reduced. Incomplete vagotomy was not responsible for this absence of change. These results are in complete contrast to those of Becker et al. (1973) who found no significant change in fasting gastrin levels after antrectomy with vagotomy and a significant increase after pyloroplasty and vagotomy. Stern and Walsh (1973) reported raised fasting serum gastrin levels in eight subjects after truncal vagotomy and pyloroplasty. However, these subjects had not been studied before the operation. In addition, several of them were not examined until some years after the operation, by which time a severe proximal gastritis might have developed, producing a more profound hypochlorhydria with resultant lack of $G$ cell suppression. Our own data confirm those of McGuigan and Trudeau (1970). We have found no significant change in the fasting serum gastrin after truncal vagotomy and pyloroplasty. Furthermore, we have shown that no significant change in fasting serum gastrin occurs as a consequence of highly selective vagotomy without any drainage procedure.

It is clear that there is considerable variation in basal gastrin levels between individuals and in the same individual when sampling is conducted in what seems to be similar conditions.

The post-prandial gastrin responses we observed seemed to be unaffected by vagotomy. Increases or decreases of peak responses occurred in different subjects after both truncal and highly selective vagotomy. These variations might be an expression 
of variable rates of postoperative recovery of homeostatic control. Clearly no hypothesis can be constructed with confidence, but later studies of the same patients, together with similar gastric biopsy studies, may help to resolve some of the confusion which at present obtains.

The absence of significant differences in fasting and post-prandial serum gastrin levels before and after either truncal vagotomy, which denervates the antrum, or highly selective vagotomy, which does not, suggests that lesser vagal activity than that occurring during an insulin test is not an important factor in the stimulation of gastrin release from the antrum. In spite of the unchanged levels of circulating gastrin after vagotomy, the acid output of the stomach is much reduced by either operation, illustrating by contrast the crucial role of the vagus in parietal-cell function. One important question is whether this inhibition of the physiological activity of the gastric parietal cell may be the first step in a process which leads eventually to atrophy of the gastric mucosa.

This study was supported in part by USPHS Grant AM 06971 and the U.S. Veterans Administration and also in part by a grant from the Cancer Research Campaign. We wish to thank Mrs Stella Linberg, Mrs Janet Constantinou, Mrs Ysanne Smart, and Mrs Elizabeth Calvert for their assistance.

\section{References}

Adams, J. F., Cox, A. G., Kennedy, E. H., and Thompson, J. (1967). Effect of medical and surgical vagotomy on intrinsic factor secretion. British Medical Journal, 3, 473-476.

Becker, H. D., Reeder, D. D., and Thompson, J. C. (1973). Effect of truncal vagotomy with pyloroplasty or with antrectomy on food-stimulated gastrin values in patients with duodenal ulcer. Surgery, 74, 580-586.

Bitsch, V., Christiansen, P. M., Faber, V., and Rødbro, P. (1966). Gastric secretory patterns before and after vagotomy. Lancet, 1, 1288-1291.

Cox, A. G., Bond, M. R., Podmore, D. A., and Rose, D. P. (1964). Aspects of nutrition after vagotomy and gastrojejunostomy. British Medical Journal, 1, 465-469.
Faber, K. (1935). Gastritis and its Consequences. Oxford University Press: London.

Findlay, J. M., Prescott, R. J., and Sircus, W. (1972). Comparative evaluation of water recovery test and fluoroscopic screening in positioning a nasogastric tube during gastric secretory studies. British Medical Journal, 4, 458461.

Gear, M. W. L., Truelove, S. C., and Whitehead, R. (1971). Gastric ulcer and gastritis. Gut, 12, 639-645.

Hollander, F. (1948). Laboratory procedures in the study of vagotomy. Gastroenterology, 11, 419-425.

Hunter, W. M., and Greenwood, F. C. (1962). Preparation of iodine-131 labelled human growth hormone of high specific activity. Nature, 194, 495-496.

McGuigan, J. E., and Trudeau, W. L. (1972). Serum gastrin levels before and after vagotomy and pyloroplasty or vagotomy and antrectomy. New England Journal of Medicine, 286, 184-188.

Rosenquist, G. L., and Holmquist, A. M. (1974). The specificity of antibodies directed to porcine gastrin. Immunochemistry, 11, 489-494.

Sanders, G. B., and Mecray, P. M. (1941). Pseudogastritis of operative origin. Annals of Surgery, 114, 986-996.

Schindler, R., Necheles, H., and Gold, R. L. (1939). Surgical gastritis. Surgery, Gynecology, and Obstetrics, 69, 281-286.

Stalsberg, H., and Taksdal, S. (1971). Stomach cancer following gastric surgery for benign conditions. Lancet, 2 , $1175-1177$.

Stern, D. H., and Walsh, J. H. (1973). Gastrin release in postoperative ulcer patients: evidence for release of duodenal gastrin. Gastroenterology, 64, 363-369.

Trudeau, W. L., and McGuigan, J. E. (1970). Serum gastrin levels in patients with peptic ulcer disease. Gastroenterology, 59, 6-12.

Wesdorp, R. I. C., and Fischer, J. E. (1974). Plasma-gastrin and acid secretion in patients with peptic ulceration. Lancet, 2, 857-860.

Whitehead, R., Roca, M., Meikle, D. D., Skinner, J. M., and Truelove, S. C. (1975). The histological classification of duodenitis in fibreoptic biopsy specimens. Digestion, 13, 129-136.

Whitehead, R., Truelove, S. C., and Gear, M. W. L. (1972). The histological diagnosis of chronic gastritis in fibreoptic gastroscope biopsy specimens. Journal of Clinical Pathology, 25, 1-11.

Yalow, R. S., and Berson, S. A. (1970). Size and charge distinctions between endogenous human plasma gastrin in peripheral blood and heptadecapeptide gastrins. Gastroenterology, 58, 609-615.

Yip, B. S. S. C., and Jordan, P. H., Jr. (1970). Radioimmunoassay of gastrin using antiserum to porcine gastrin 1 (34796). Proceedings of the Society for Experimental Biology and Medicine, 134, 380-385. 